\title{
openheart Rates and risk of arrhythmias in cancer survivors with chemotherapy-induced cardiomyopathy compared with patients with other cardiomyopathies
}

\author{
Michael G Fradley, ${ }^{1,2}$ Federico Viganego, ${ }^{1,2}$ Kevin Kip, ${ }^{3}$ Angel Martin, ${ }^{4}$ \\ Aarti A Patel, ${ }^{1}$ Roohi Ismail-Khan, ${ }^{2,5}$ Sanders Chae, ${ }^{1}$ Bengt Herweg, \\ Arthur Labovitz
}

To cite: Fradley MG, Viganego $F$ Kip K, et al. Rates and risk of arrhythmias in cancer survivors with chemotherapy-induced cardiomyopathy compared with patients with other cardiomyopathies. Open Heart 2017;4:e000701. doi:10.1136/ openhrt-2017-000701

Received 17 August 2017 Revised 17 November 2017 Accepted 27 November 2017

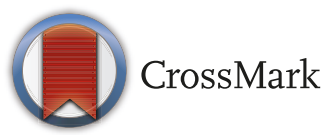

${ }^{1}$ Division of Cardiovascular Medicine, University of South Florida, Tampa, Florida, USA ${ }^{2}$ Cardio-Oncology Program, H Lee Moffitt Cancer Center and Research Institute, Tampa, Florida, USA

${ }^{3}$ Department of Epidemiology and Biostatistics, University of South Florida College of Public Health, Tampa, Florida, USA

${ }^{4}$ Department of Internal Medicine, USF Health Morsani College of Medicine, Tampa Florida, USA

${ }^{5}$ Women's Oncology Program, H Lee Moffitt Cancer Center and Research Institute, Tampa, Florida, USA

Correspondence to Dr Michael G Fradley; mfradley@health.usf.edu

\section{ABSTRACT}

Objectives There is little information about arrhythmia burden in cancer survivors with chemotherapy-induced cardiomyopathy $(\mathrm{CIC})$. We hypothesise that the rates and risk of arrhythmias will be similar in $\mathrm{CIC}$ when compared with other non-ischaemic cardiomyopathy (NICMO) aetiologies.

Methods We retrospectively identified nine patients with $\mathrm{CIC}$ and an implantable defibrillator and 18 age and sex-matched control patients (nine patients with NICMO and nine patients with ischaemic cardiomyopathy (ICMO)). Rates and odds of arrhythmias were calculated by type of cardiomyopathy, adjusting for days since implantable cardioverter defibrillator implantation, history of atrial fibrillation and length of follow-up using logistic regression analysis.

Results Compared with patients with NICM0, rates and adjusted odds were similar for patients with $\mathrm{CIC}$ for atrial arrhythmias (44.4\% vs $33.3 \%$; adjusted $\mathrm{OR}=1.89$; $95 \% \mathrm{Cl} 0.17$ to $21.03 ; \mathrm{P}=0.61)$, non-sustained ventricular tachycardia (NSVT) $(44.4 \%$ vs $33.3 \% ; 0 R=2.10 ; 95 \% \mathrm{Cl}$ 0.21 to 20.56; $P=0.53$ ), and the combined outcome of NSVT, sustained ventricular tachycardia and/or ventricular fibrillation (44.4\% vs $44.4 \%$; OR=2.70; $95 \% \mathrm{Cl} 0.25$ to 29.48; $\mathrm{P}=0.42$ ). Conversely, compared with patients with NICMO, patients with ICMO demonstrated higher rates and adjusted odds of the combined outcome $(88.9 \%$ vs 44.4\%; $\mathrm{OR}=28.60 ; 95 \% \mathrm{Cl} 1.26$ to $648.2 ; \mathrm{P}=0.04$ ) and NSVT $(77.8 \%$ vs $33.3 \%$; $\mathrm{OR}=8.95 ; 95 \% \mathrm{Cl} 0.90$ to 88.94 $\mathrm{P}=0.06)$.

Conclusions While tentative based on sample size, rates of arrhythmias in patients with $\mathrm{CIC}$ appear to be similar to those experienced by patients with other forms of NICMO.

\section{INTRODUCTION}

Over the last decade, there has been significant improvement in the survival rates of many cancers. This has been attributed to advances in screening and the development and use of novel therapeutics. As a result, it is estimated that more than 14 million cancer survivors are living in the USA. ${ }^{1}$ Despite these improvements, there is also an increasing prevalence of treatment-related cardiovascular

\section{KEY QUESTIONS}

What is already known about this subject? There are little data regarding the frequency of arrhythmias or implantable cardioverter defibrillator therapies and outcomes in patients with chemotherapy-induced cardiomyopathy. One study demonstrated non-sustained ventricular tachycardia was the most common rhythm disturbance in patients with anthracycline-mediated cardiomyopathy, followed by atrial fibrillation/flutter and sustained ventricular tachycardia or fibrillation at rates similar to those observed in patients with other types of cardiomyopathy.

\section{What does this study add?}

Given the paucity of information on this topic, this study adds additional data regarding arrhythmia burden in patients with chemotherapy-induced cardiomyopathy. Our data suggest that arrhythmias in this population are not substantially increased compared with other types of non-ischaemic cardiomyopathies and are not likely to cause significantly increased morbidity or mortality.

How might this impact on clinical practice? It was previously felt that chemotherapy-induced cardiomyopathy was associated with worse outcomes compared with other aetiologies thus affecting treatment decisions. These data suggest that patients with chemotherapy-induced cardiomyopathy have arrhythmia burdens similar to other patients with non-ischaemic cardiomyopathy. As such, they should receive the same evidence-based therapies that would be offered to patients with other forms of cardiomyopathy.

(CV) complications, which is a key predictor of outcomes among these patients. ${ }^{2}$ In cancer survivors, cardiomyopathy and heart failure (HF) are among the most common long-term cardiotoxicities associated with different cancer treatments including anthracyclines and targeted therapies. ${ }^{3} 4$ Although 
anthracycline-induced cardiomyopathy (AIC) may develop relatively quickly after exposure, delayed effects decades after receiving these cardiotoxic agents are well established. ${ }^{35}$ Historically, a diagnosis of AIC was thought to portend a worse prognosis than other forms of cardiomyopathy. ${ }^{6}$ Patients who develop AIC may present with a broad spectrum of clinical manifestations ranging from asymptomatic left ventricular (LV) dysfunction, decompensated HF or arrhythmias. ${ }^{3}$ In addition, these patients are also at increased risk of sudden cardiac death (SCD) primarily from ventricular tachyarrhythmias. ${ }^{7-9}$ Implantable cardioverter defibrillators (ICD) have been shown in several multicentre trials to provide a significant survival benefit, and are recommended for primary prevention according to the Heart Rhythm Society/American Heart Association/American College of Cardiology guidelines in patients with left ventricular ejection fraction (LVEF) $\leq 35 \%$ despite adequate medical therapy, regardless of aetiology, ${ }^{10}$ though recent studies have questioned the benefit of these devices in patients with non-ischaemic cardiomyopathies. ${ }^{11}$

Besides providing protection from life-threatening ventricular arrhythmias, ICDs store information about the type and frequency of arrhythmias. This information is acquired from device interrogations at routine visits or via remote telemetry monitoring from the patient's home. To date, few studies have been published evaluating the frequency of arrhythmias or ICD therapies and outcomes in patients with chemotherapy-induced cardiomyopathy (CIC) ${ }^{12}$ The purpose of our study was to examine the prevalence of atrial and ventricular arrhythmias in patients with CIC treated with ICDs compared with a matched population of patients with either ischaemic cardiomyopathy (ICMO) or other types of non-ischaemic cardiomyopathy (NICMO).

\section{METHODS}

All procedures performed in studies involving human participants were in accordance with the ethical standards of the institutional and/or national research committee and with the 1964 Declaration of Helsinki and its later amendments or comparable ethical standards. This was a retrospective chart review study. As such, informed consent is not necessary. The patient population was derived from the University of South Florida (USF) Electrophysiology Service device clinic. Patient and device-related data are collected and maintained in the electronic medical record for all outpatient clinic visits and remote device interrogations. All patients followed in the USF device clinic from 2005 to 2015 for at least 90 days post-ICD or cardiac resynchronization therapy defibrillator (CRT-D) implantation were considered for inclusion. All patients received cardiac implantable electronic devices (CIED) in accordance with published guidelines. ${ }^{10}$ Patients were considered to have a CIC if they had a history of cancer and developed cardiac dysfunction (decrease in the LVEF of $>10$ percentage points, to a value $<53 \%$ ) after exposure to chemotherapy known to cause cardiomyopathy and HF, without an obvious alternative aetiology (ie, underlying coronary artery disease was excluded with stress testing and/or coronary angiography, and so on). This definition is consistent with those published by the American Society of Echocardiography and the European Society of Cardiology. ${ }^{13} 14$ These patients were then compared with a cohort of age and sex-matched patients with NICMO (not due to chemotherapy) and second cohort of age and sex-matched patients with ICMO (with known obstructive coronary artery disease). Individual matching was performed, with mean age within 5 years across the respective groups. Classification of cardiomyopathy is based on accepted societal recommendations including objective evidence of significant coronary artery disease for all patients diagnosed with ICMO. Inclusion criteria included implantation of either an ICD or CRT-D for primary prevention purposes, baseline ejection fraction (EF) of $\leq 35 \%$ and appropriate follow-up as outlined above. For the NICMO cohort, only those patients with dilated cardiomyopathies were included. All patients in this group had undergone stress testing and/or coronary angiography to exclude the possibility of underlying coronary artery disease. Patients with a lack of appropriate minimum follow-up, patients lacking adequate device interrogation data, patients with devices implanted for secondary prevention purposes (ie, ventricular tachycardia (VT) or ventricular fibrillation (VF) at baseline) and patients with high-risk arrhythmic substrates (ie, hypertrophic cardiomyopathy, arrhythmogenic right ventricular cardiomyopathy) were excluded.

Medical records of patients who met inclusion criteria were thoroughly evaluated for baseline characteristics, including age, sex, pre-existing CV risk factors or disease (hypertension, hyperlipidaemia, coronary artery disease, diabetes, atrial fibrillation (AF), chronic kidney disease, stroke), smoking history, body mass index, CV medications (beta blockers, statins, ACE inhibitors/angiotensin receptor blockers, diuretics, digoxin, antiarrhythmics), cancer type, chemotherapy regimen, baseline EF and indication for device implantation. The number of arrhythmic events and the type of ICD therapy were evaluated from all available device interrogation reports. All recorded arrhythmia ECGs were reviewed and appropriately adjudicated by electrophysiologists. Device programming was determined by the implanting and/or treating electrophysiologist.

Descriptive statistics are presented as mean and SD for continuous variables and frequencies for categorical variables. To compare baseline characteristics between the three study cohorts (type of cardiomyopathy), Student's t-tests and analysis of variance were used for continuous variables; Fisher's exact test of proportions was used for categorical variables. Initially, crude event rates of atrial and ventricular arrhythmias were calculated along with ORs calculated by use of logistic regression analysis with the non-ischaemic group serving as the reference 
category. Recognising limited sample size, adjusted ORs and 95\% CIs were calculated with adjustment for the three important variables: days since ICD implant, history of AF and length of follow-up. The latter variable was particularly important in that precise time-to-event data were not available for outcomes of interest and length of follow-up (ie, at-risk period) differed between the three groups. Finally, event rates of antitachycardia pacing (ATP) and defibrillation/shock were compared by type of cardiomyopathy by use of Fisher's exact test of proportions. A two-sided $\mathrm{P}$ value of $\leq 0.05$ was used to define statistical significance.

\section{RESULTS}

In addition to the 18 matched subjects (NICMO=9, ICMO=9), a total of nine patients met inclusion criteria in the CIC study cohort. Among the CIC cohort, all patients had a diagnosis of breast cancer or leukaemia/ lymphoma. The mean time from chemotherapy exposure to device implantation was 12.7 years (range 2-20 years). The majority of patients were exposed to anthracycline-based chemotherapy regimens $(78 \%)$ with the remaining patients receiving other regimens including those with cyclophosphamide. Comparing the three study cohorts, coronary artery disease and diabetes were expectedly more common in the ICMO group compared with patients with CIC or NICMO, and patients with CIC were less likely to report a history of smoking; however, other baseline patient characteristics did not vary significantly between groups (table 1). Consequently, statin use was also more common in the ICMO group; however, the use of other CV medications was similar between the groups. Specifically, there was no difference in the use of antiarrhythmic medications at baseline, with only one patient in the ICMO group treated with amiodarone. Over the course of follow-up, antiarrhythmics were not initiated in any additional patients among the three groups. There was one patient with CIC with non-obstructive coronary artery disease which was not felt to have contributed to his LV dysfunction. No patients in any cohort had greater than grade 1 hypertension. ${ }^{15}$ Baseline arrhythmias (AF, sustained VT or VF) did not differ between groups. Mean baseline LVEF (assessed by echocardiography in 25 patients $(92.6 \%)$ and cardiac MRI in the remaining 2 patients (7.4)) did not differ significantly between groups (CIC 29.8\%; NICMO 22.8\%; ICMO 27.0\%; $\mathrm{P}=0.23$ ).

In our cohort, patients with CIC and other patients with NICMO were more likely to receive a biventricular ICD than patients with ICMO $(66.7 \%, 55.6 \%, 11.1 \% ; \mathrm{P}=0.05)$. Mean follow-up duration in days for the CIC, NICMO and ICMO cohorts was 1052, 1826 and 1298, respectively $(\mathrm{P}=0.08)$. Thus, the reference group of patients with

Table 1 Baseline characteristics of study participants

\begin{tabular}{lllll}
\hline & $\begin{array}{l}\text { Non-ischaemic } \\
\text { cardiomyopathy }\end{array}$ & $\begin{array}{l}\text { In=9) } \\
\text { Ischaemic cardiomyopathy } \\
(\mathbf{n = 9}\end{array}$ & $\begin{array}{l}\text { Chemotherapy-induced } \\
\text { cardiomyopathy }(\mathbf{n}=9)\end{array}$ & P value \\
\hline Age (years) & $49.4 \pm 13.8$ & $54.3 \pm 12.2$ & $49.1 \pm 13.5$ & 0.65 \\
\hline Female & 77.8 & 77.8 & 77.8 & 1.0 \\
\hline BMI $\left(\mathrm{kg} / \mathrm{m}^{2}\right)$ & $28.8 \pm 6.3$ & $32.5 \pm 8.6$ & $32.3 \pm 7.4$ & 0.50 \\
\hline LVEF & $22.8 \pm 7.3$ & $27.0 \pm 7.0$ & $29.8 \pm 10.6$ & 0.23 \\
\hline Hypertension & 55.6 & 77.8 & 66.7 & 0.87 \\
\hline CAD & 0.0 & $100.0^{*}$ & 11.1 & 0.001 \\
\hline Diabetes & 11.1 & $77.8^{*}$ & 22.2 & 0.02 \\
\hline Atrial fibrillation & 0.0 & 22.2 & 22.2 & 0.50 \\
\hline Sustained VT/NF & 0.0 & 0.0 & 0.0 & 1.0 \\
\hline CVA & 11.1 & 33.3 & 22.2 & 0.84 \\
\hline CKD & 11.1 & 11.1 & 11.1 & 1.0 \\
\hline Smoking history & 66.7 & 66.7 & $0.0 \dagger$ & 0.006 \\
\hline Medications & & & & 1.0 \\
\hline Beta blocker & 100.0 & 100.0 & 88.9 & 0.05 \\
\hline Statin & 33.3 & $88.9^{*}$ & 44.4 & 0.57 \\
\hline ACE--//ARB & 88.9 & 88.9 & 66.7 & 1.0 \\
\hline Digoxin & 33.3 & 33.3 & 44.4 & 0.35 \\
\hline Antiarrhythmic & 0.0 & 11.1 & 0.0 & \\
\hline
\end{tabular}

Values are mean \pm SD or per cent.

${ }^{*} \mathrm{P}<0.05$ comparing non-ischaemic versus ischaemic cardiomyopathy subjects.

$\dagger \mathrm{P}<0.05$ comparing non-ischaemic versus chemotherapy-induced cardiomyopathy subjects.

ACE-I, ACE inhibitor; ARB, angiotensin receptor blocker; BMI, body mass index; CAD, coronary artery disease; CKD, chronic kidney disease;

CVA, cerebrovascular attack; LVEF, left ventricular ejection fraction; VF, ventricular fibrillation; VT, ventriculartachycardia. 


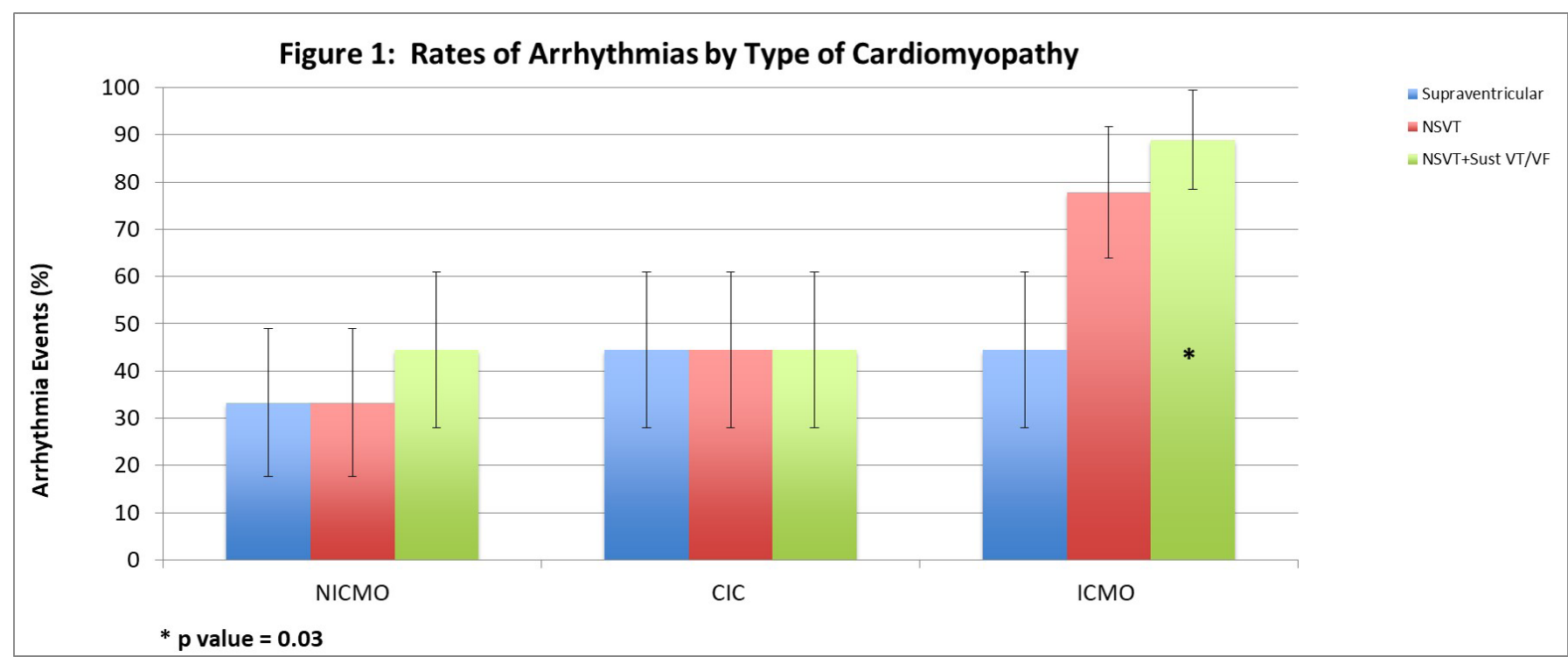

Figure 1 Rates of arrhythmias by type of cardiomyopathy. * $\mathrm{P}=0.03$. $\mathrm{CIC}$, chemotherapy-induced cardiomyopathy; ICMO, ischaemic cardiomyopathy; NICMO, non-ischaemic cardiomyopathy; NSVT, non-sustained ventricular tachycardia; VF, ventricular fibrillation; VT, ventricular tachycardia.

NICMO had a somewhat longer period of follow-up as compared with the CIC and ICMO cohorts. Adjusting for days since ICD implantation, history of AF and length of follow-up, and as compared with patients with NICMO, rates and adjusted ORs were generally similar for patients with CIC for atrial arrhythmias (44.4\% vs $33.3 \%$; adjusted $\mathrm{OR}=1.89 ; 95 \%$ CI 0.17 to $21.03 ; \mathrm{P}=0.73$ ), non-sustained ventricular tachycardia (NSVT) $(44.4 \%$ vs $33.3 \%$; adjusted $\mathrm{OR}=2.10 ; 95 \%$ CI 0.21 to $20.56 ; \mathrm{P}=0.53$ ) and the combined outcome of NSVT, sustained VT and/ or VF ( $44.4 \%$ vs $44.4 \%$; adjusted $\mathrm{OR}=2.70 ; 95 \%$ CI 0.25 to 29.48; $\mathrm{P}=0.42)$. In contrast, compared with patients with NICMO, patients with ICMO demonstrated higher rates and adjusted odds of the combined outcome $(88.9 \%$ vs $44.4 \%$; adjusted $\mathrm{OR}=28.60 ; 95 \%$ CI 1.26 to $648.2 ; \mathrm{P}=0.04$ ) and NSVT $(77.8 \%$ vs $33.3 \%$; adjusted OR=8.95; $95 \%$ CI 0.90 to $88.94 ; \mathrm{P}=0.06$ ). There was no significant difference in atrial arrhythmias between these groups, however (figure 1; table 2). Finally, comparing patients with CIC with patients with ICMO who had similar length of follow-up, the rate and risk for the combined outcome was non-significantly lower in the CIC group ( $44.4 \%$ vs $88.9 \%$, adjusted $\mathrm{OR}=0.19,95 \%$ CI 0.10 to $3.59, \mathrm{P}=0.27)$.

There were non-significant differences in the rates of ICD therapies (either appropriate shocks or ATP) among the three groups (table 3 ). There were also no significant

Table 2 Event rates and odds of events by type of cardiomyopathy $(n=27)^{\star}$

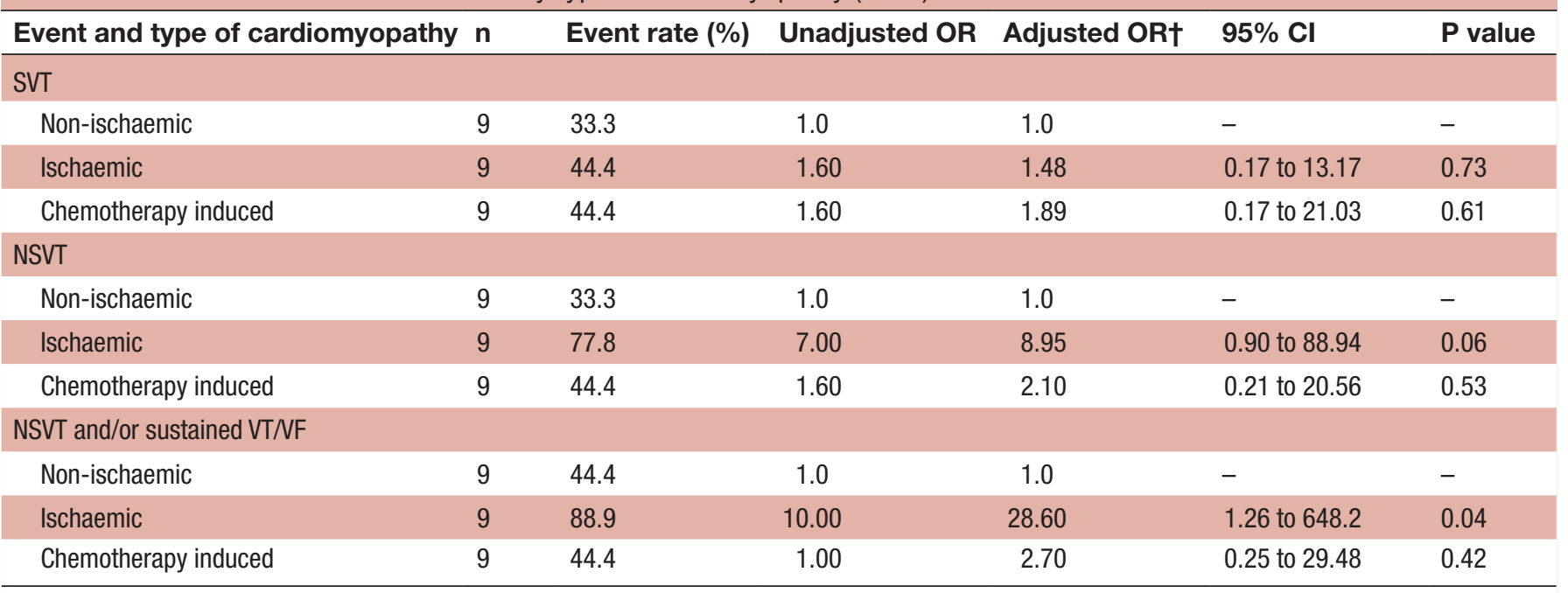

*Non-ischaemic cardiomyopathy used as the comparator group.

†Adjusted for days since implantable cardioverter defibrillator (ICD) implant, history of atrial fibrillation and length of follow-up.

NSVT, non-sustained ventricular tachycardia; SVT, supraventricular tachycardia (includes atrial fibrillation); VF, ventricular fibrillation; VT, ventricular tachycardia. 
Table 3 Event rates of appropriate ICD therapies by type of cardiomyopathy $(n=27)$

\begin{tabular}{lccc}
\hline $\begin{array}{l}\text { ICD therapy and type of } \\
\text { cardiomyopathy }\end{array}$ & $\mathbf{n}$ & Event rate (\%) & P value \\
\hline $\begin{array}{l}\text { Antitachycardia pacing (ATP) } \\
\quad \text { Non-ischaemic }\end{array}$ & 9 & 11.1 & 1.0 \\
\hline $\begin{array}{l}\text { Ischaemic } \\
\quad \text { Chemotherapy induced }\end{array}$ & 9 & 11.1 & \\
\hline $\begin{array}{l}\text { Defibrillation/shock } \\
\quad \text { Non-ischaemic }\end{array}$ & 9 & 0.0 & \\
\hline $\begin{array}{l}\text { Ischaemic } \\
\text { Chemotherapy induced }\end{array}$ & 9 & 11.1 & \\
\hline
\end{tabular}

ICD, implantable cardioverter defibrillator.

differences in the rates of inappropriate shocks between each group (CIC 11.1\%; NICMO 0\%; ICMO 22.2\%; $\mathrm{P}=0.75)$. Patients with CIC and NICMO were more likely to be programmed with only one therapy zone rather than two or more zones (CIC 77.8\%; NICMO $66.7 \%$; ICMO $33.3 \% ; \mathrm{P}=0.23)$. In addition, over the course of the follow-up period, there were no reported deaths in any cohort.

\section{DISCUSSION}

While CIC is a well-recognised entity, there is a relative paucity of information regarding arrhythmia burden in this population. The current study evaluated the rates and risk of arrhythmias in patients with CIC compared with patients with other forms of NICMO and ICMO. When adjusting for days since ICD implantation, history of arrhythmias including AF and length of follow-up, the rates and adjusted odds for atrial arrhythmias, NSVT and the combined outcome of NSVT, sustained VT and/or VF were generally similar in patients with CIC compared with other forms of NICMO. In contrast, patients with ICMO had higher rates and adjusted odds of both the combined outcome as well as NSVT. There were no significant differences in rates of appropriate ICD therapies (either shocks or ATP) between the three groups. Despite the limited sample size, these data are consistent with recently published results demonstrating the burden of arrhythmias in patients with CIC is similar to other forms of cardiomyopathy. ${ }^{12}$

With improved screening and treatment options, patients are living longer and in many cases, surviving their cancer. Despite the improvements in cancer-specific outcomes, CV toxicities are frequent complications of these therapies, adversely affecting both patient and survivor mortality and quality of life. ${ }^{16}$ Among childhood cancer survivors, CV disease is the third most common cause of death, behind cancer recurrence or the development of a second malignancy. These patients have a striking sevenfold increased risk of CV death compared with the general population. ${ }^{17}$ In addition, the cumulative incidence of HF by age 45 has been reported at
$4.8 \%$, while the risk of arrhythmias in that population is only $1.3 \%$, with the majority of these patients exposed to anthracyclines and/or radiation therapy. ${ }^{18}$ Rates of anthracyclines-associated $\mathrm{HF}$ vary from $5 \%$ to $26 \%$ depending on the population studied. ${ }^{5}$ For example, the risk of developing HF among survivors exposed to more than $300 \mathrm{mg} / \mathrm{m}^{2}$ of anthracycline has been reported at more than $10 \%$ by 20 years after exposure. ${ }^{19}$ In addition to high cumulative dose, anthracycline-mediated cardiotoxicity is more common at the age extremes, in those with pre-existing $\mathrm{CV}$ disease and in women. ${ }^{20}$ Indeed, our data supports these observations. The mean age in our study was 49 years (range $26-70$ ), with $78 \%$ receiving anthracyclines. The median time from chemotherapy to device implantation was 13 years, and $77.8 \%$ of patients were women, consistent with historical findings.

It has been traditionally thought that AIC portends a worse prognosis when compared with other forms of cardiomyopathy. ${ }^{6}$ The exact cause of the increased mortality has not been determined but potential theories include progression/recurrence of cancer, pump failure or malignant arrhythmias. Furthermore, many of these patients do not receive optimal HF management. In one study evaluating patients with asymptomatic LV dysfunction in the setting of chemotherapy, only $31 \%$ of patients received angiotensin system inhibitor therapy and $35 \%$ were treated with a beta blocker. ${ }^{21}$ In our study, there was no statistically significant difference in the medical therapies offered to the patients with the exception of statins which were expectedly more frequently administered to those with ICMO. While this suggests patients with significant CIC are now being treated with appropriate medications, our data do not clarify the timing at which these medications were initiated and may have only been started after serious LV dysfunction and HF were identified.

There is increasing recognition that arrhythmias are a common CV complication affecting patients with cancer. Atrial arrhythmias, particularly AF, can occur with increased frequency in patients with cancer, regardless of their treatment exposures. ${ }^{22-24}$ In addition, multiple chemotherapies, targeted therapies and immunotherapies have been associated with increased arrhythmia burden, often a result of a primary toxicity such as myocardial dysfunction, ischaemia or inflammation leading to an arrhythmic substrate. ${ }^{25-27}$ Rhythm disturbances related to anthracycline exposure have been well established. For example, up to $10 \%$ of patients treated with doxorubicin will develop AF after the first cycle. ${ }^{28}$ Premature ventricular contractions have been reported in up to $30 \%$ of patients receiving anthracyclines with $6 \%$ developing NSVT, while sustained ventricular arrhythmias are relatively rare. ${ }^{28-31}$ The exact mechanism of anthracycline-induced arrhythmias remains unclear; however, proposed theories include effects on the L-type $\mathrm{Ca}^{2+}$ channels, impaired intracellular signalling or the accumulation of toxic metabolites. ${ }^{26} 32$ A recent study evaluating 209 patients with active cancer with an ICD demonstrated an increased risk of ventricular arrhythmias, particularly 
if metastatic disease was present. ${ }^{33}$ There are less data evaluating the risk of arrhythmias in cancer survivors including those who have developed CIC; however, these findings are particularly relevant given the potentially worse outcomes in patients with this form of LV dysfunction. ${ }^{891234}$ In one study using Holter monitors to evaluate survivors of paediatric cancers with normal LV function, $3 \%$ of patients demonstrated NSVT. ${ }^{34}$ In contrast, a different study evaluating 15 paediatric survivors with AIC reported arrhythmias in 14 patients, with three patients experiencing SCD and one patient receiving an ICD. ${ }^{9}$

It can be particularly challenging to accurately assess arrhythmia burden in patients. As such, individuals with CIEDs represent an ideal population to evaluate given that these devices continuously record and store arrhythmia data. In our study, we systematically evaluated data from patients with CIC and a CIED and compared the findings with age and sex-matched control patients with other forms of NICMO or ICMO. Our pilot study suggests relatively similar rates and adjusted odds for atrial arrhythmias, NSVT and VT/VF when comparing CIC with other forms of NICMO; however, there were higher rates and adjusted odds for both the combined outcome and NSVT in the ICMO group. These data support the findings established by Mazur and colleagues in their recently published study that demonstrated NSVT was the most common rhythm disturbance in patients with anthracycline-mediated cardiomyopathy, followed by $\mathrm{AF} /$ flutter and sustained VT/VF at rates similar to those observed in their two comparator groups. Moreover, there was no difference in clinical outcomes including overall mortality or heart transplantation among the groups. ${ }^{12}$ Our data in combination with Mazur et al suggest arrhythmias in the CIC population are not substantially increased and are not likely to cause significantly increased morbidity or mortality.

Our study has several limitations however. It is an observational (non-randomised) retrospective analysis with associated inherent weaknesses, in particular, the ability to fully control for potential confounding. Although the data were abstracted from a small sample size which impacts precision and may affect generalisability, developing a CIC and requiring an implantable defibrillator is a rare phenomenon and the number of included patients and baseline demographics is similar to those in previously published studies. Moreover, evaluating patients with a diagnosis of CIC and concurrent implantable device may introduce selection bias. Although we quantified arrhythmia burden, the study was not designed to evaluate CV outcomes including HF hospitalisation or mortality, nor can we comment on the overall benefit of ICDs in this population. Prospective multicentre studies, including the Multicenter Automatic Defibrillator Implantation Trial-Chemotherapy-Induced Cardiomyopathy trial (NCT02164721), are necessary to further and more completely answer these questions. Finally, the length of follow-up (at-risk period) was longer in the NICMO group which was used as the reference group for comparisons with patients with CIC and ICMO. While we adjusted for this differential length of follow-up in the analysis, results should be cautiously interpreted, especially given the small sample size.

\section{CONCLUSIONS}

Rates of atrial and ventricular arrhythmias in cancer survivors with CIC appear to be similar to those experienced by patients with other forms of NICMO, and lower than patients with ICMO. Despite the small sample size, this study provides additional evidence to our relatively limited knowledge about the type and impact of arrhythmias on cancer survivors with significant CIC. The development of future prospective multicentre trials will allow for the evaluation of a larger sample population leading to more definitive data regarding rhythm disturbances in patients with CIC.

Contributors MGF: designed the study; collected and analysed the data; wrote, reviewed and edited the manuscript. FV: collected and analysed the data; reviewed and edited the manuscript. KK: developed the statistical analysis plan; analysed the data; wrote, reviewed and edited the manuscript. AM: collected the data; wrote, reviewed and edited the manuscript. AAP, RIK, SC, BH, AL: reviewed and edited the manuscript.

Competing interests None declared.

Ethics approval The study was approved by the University of South Florida (USF) Institutional Review Board and complies with all standards set forth by the university.

Provenance and peer review Not commissioned; externally peer reviewed.

Data sharing statement There are no additional data available for this paper.

Open Access This is an Open Access article distributed in accordance with the Creative Commons Attribution Non Commercial (CC BY-NC 4.0) license, which permits others to distribute, remix, adapt, build upon this work non-commercially, and license their derivative works on different terms, provided the original work is properly cited and the use is non-commercial. See: http://creativecommons.org/ licenses/by-nc/4.0/

(c) Article author(s) (or their employer(s) unless otherwise stated in the text of the article) 2017. All rights reserved. No commercial use is permitted unless otherwise expressly granted.

\section{REFERENCES}

1. Miller KD, Siegel RL, Lin CC, et al. Cancer treatment and survivorship statistics, 2016. CA Cancer J Clin 2016;66:271-89.

2. Reulen RC, Winter DL, Frobisher C, et al. Long-term causespecific mortality among survivors of childhood cancer. JAMA 2010;304:172-9.

3. Yeh ET, Bickford CL. Cardiovascular complications of cancer therapy: incidence, pathogenesis, diagnosis, and management. J Am Coll Cardiol 2009;53:2231-47.

4. Yu AF, Steingart RM, Fuster V. Cardiomyopathy associated with cancer therapy. J Card Fail 2014;20:841-52.

5. Swain SM, Whaley FS, Ewer MS. Congestive heart failure in patients treated with doxorubicin: a retrospective analysis of three trials. Cancer 2003;97:2869-79.

6. Felker GM, Thompson RE, Hare JM, et al. Underlying causes and long-term survival in patients with initially unexplained cardiomyopathy. N Engl J Med 2000;342:1077-84.

7. Goldman S, Johnson G, Cohn JN, et al. Mechanism of death in heart failure. The vasodilator-heart failure trials. The V-HeFT VA cooperative studies group. Circulation 1993;87(6 Suppl):VI24-31.

8. Rudzinski T, Ciesielczyk M, Religa W, et al. Doxorubicin-induced ventricular arrhythmia treated by implantation of an automatic cardioverter-defibrillator. Europace 2007;9:278-80.

9. Steinherz LJ, Steinherz PG, Tan C. Cardiac failure and dysrhythmias 6-19 years after anthracycline therapy: a series of 15 patients. Med Pediatr Oncol 1995;24:352-61.

10. Epstein AE, DiMarco JP, Ellenbogen KA, et al. ACC/AHA/HRS 2008 guidelines for device-based therapy of cardiac rhythm abnormalities: a report of the American college of cardiology/American heart 
association task force on practice guidelines (writing committee to revise the acc/aha/naspe 2002 guideline update for implantation of cardiac pacemakers and antiarrhythmia devices): developed in collaboration with the American association for thoracic surgery and society of thoracic surgeons. Circulation 2008;117:e350-408.

11. Køber L, Thune JJ, Nielsen JC, et al. Defibrillator implantation in patients with nonischemic systolic heart failure. $N$ Engl J Med 2016;375:1221-30.

12. Mazur M, Wang F, Hodge DO, et al. Burden of cardiac arrhythmias in patients with anthracycline-related cardiomyopathy. JACC Clin Electrophysiol 2017;3:139-50.

13. Plana JC, Galderisi M, Barac A, et al. Expert consensus for multimodality imaging evaluation of adult patients during and after cancer therapy: a report from the American society of echocardiography and the european association of cardiovascular imaging. J Am Soc Echocardiogr 2014;27:911-39.

14. Zamorano JL, Lancellotti P, Rodriguez Muñoz D, et al. 2016 ESC Position Paper on cancer treatments and cardiovascular toxicity developed under the auspices of the ESC committee for practice guidelines: the task force for cancer treatments and cardiovascular toxicity of the European Society of Cardiology (ESC). Eur Heart $J$ 2016;37:2768-801.

15. Chobanian AV, Bakris GL, Black HR, et al. The seventh report of the joint national committee on prevention, detection, evaluation, and treatment of high blood pressure: the JNC 7 report. JAMA 2003;289:2560-72.

16. Nathan PC, Amir E, Abdel-Qadir H. Cardiac outcomes in survivors of pediatric and adult cancers. Can J Cardiol 2016;32:871-80.

17. Mertens AC, Liu Q, Neglia JP, et al. Cause-specific late mortality among 5-year survivors of childhood cancer: the childhood cancer survivor study. J Natl Cancer Inst 2008;100:1368-79.

18. Armstrong GT, Oeffinger $\mathrm{KC}$, Chen $\mathrm{Y}$, et al. Modifiable risk factors and major cardiac events among adult survivors of childhood cancer. $J$ Clin Oncol 2013;31:3673-80.

19. van Dalen EC, van der Pal HJ, Kok WE, et al. Clinical heart failure in a cohort of children treated with anthracyclines: a long-term followup study. Eur J Cancer 2006;42:3191-8.

20. Chow EJ, Chen Y, Kremer LC, et al. Individual prediction of heart failure among childhood cancer survivors. J Clin Oncol 2015;33:394-402.
21. Yoon GJ, Telli ML, Kao DP, et al. Left ventricular dysfunction in patients receiving cardiotoxic cancer therapies are clinicians responding optimally? J Am Coll Cardiol 2010;56:1644-50.

22. Farmakis D, Parissis J, Filippatos G. Insights into onco-cardiology: atrial fibrillation in cancer. J Am Coll Cardiol 2014;63:945-53.

23. Guzzetti S, Costantino G, Vernocchi A, et al. First diagnosis of colorectal or breast cancer and prevalence of atrial fibrillation. Intern Emerg Med 2008;3:227-31.

24. O'Neal WT, Lakoski SG, Qureshi W, et al. Relation between cancer and atrial fibrillation (from the reasons for geographic and racial differences in stroke study). Am J Cardiol 2015;115:1090-4.

25. Viganego F, Singh R, Fradley MG. Arrhythmias and other electrophysiology issues in cancer patients receiving chemotherapy or radiation. Curr Cardiol Rep 2016;18:52.

26. Tamargo J, Caballero R, Delpón E. Cancer chemotherapy and cardiac arrhythmias: a review. Drug Saf 2015;38:129-52.

27. Guglin M, Aljayeh M, Saiyad S, et al. Introducing a new entity: chemotherapy-induced arrhythmia. Europace 2009;11:1579-86.

28. Kilickap S, Barista I, Akgul E, et al. Early and late arrhythmogenic effects of doxorubicin. South Med J 2007;100:262-5.

29. Steinberg JS, Cohen AJ, Wasserman AG, et al. Acute arrhythmogenicity of doxorubicin administration. Cancer 1987;60:1213-8.

30. O'Bryan RM, Luce JK, Talley RW, et al. Phase II evaluation of adriamycin in human neoplasia. Cancer 1973;32:1-8.

31. Outomuro D, Grana DR, Azzato F, et al. Adriamycin-induced myocardial toxicity: new solutions for an old problem? Int $J$ Cardiol 2007;117:6-15

32. Earm YE, Ho WK, So I. Effects of adriamycin on ionic currents in single cardiac myocytes of the rabbit. $\mathrm{J} \mathrm{Mol} \mathrm{Cell} \mathrm{Cardiol}$ 1994;26:163-72.

33. Enriquez A, Biagi J, Redfearn D, et al. Increased incidence of ventricualr arrhythmias in patients with advanced cancer and implantable cardioverter-defibrillators. J Am Coll Cardiol EP 2017;3:50-6.

34. Larsen RL, Jakacki RI, Vetter VL, et al. Electrocardiographic changes and arrhythmias after cancer therapy in children and young adults. Am J Cardiol 1992;70:73-7. 